\title{
МЕТОДЫ ПРЕДСКАЗАНИЯ МНОГОКОМПОНЕНТНЫХ АЗЕОТРОПОВ
}

\author{
(Представил О. Эйзен)
}

Образование азеотропов, их тип и свойства определяют структуру диаграмм фазовых равновесий жидкость-пар, жидкость-жидкость-пар и особенности протекания этих фазовых процессов в смесях. Характеристики азеотропов учитываются при расчете параметров разделительной аппаратуры и построении технологических схем разделения и очистки веществ. Все это сделало азеотропы предметом многих теоретических и экспериментальных исследований. Ряд фундаментальных положений термодинамики растворов, установленных Дж. У. Гиббсом, Д. П. Коноваловым, М. С. Вревским и получивших дальнейшее развитие в трудах современных ученых, связан с явлением азеотропии. На практическом использовании азеотропов основаны специальные методы ректификации - азеотропная и азеотропно-экстрактивная [1, 2$]$.

K настоящему времени накоплен и систематизирован в специальных справочниках большой экспериментальный материал о свойствах азеотропов, который постоянно пополняется и уточняется. Наряду с этим усилия исследователей в области изучения азеотропии направлены на разработку методов расчета характеристик азеотропных смесей, образуемых различным сочетанием компонентов системы. Стремление заменить экспериментальное определение свойств азеотропных смесей расчетным можно объяснить тем, что расчет дает возможность, с одной стороны, обойти технические трудности опытного выделения азеотропов и измерения их параметров, которые возрастают с увеличением числа компонентов, а с другой - отобрать из числа еще не изученных азеотропов те, которые обладают нужными свойствами. Наибольшие успехи достигнуты в разработке методов расчета характеристик многокомпонентных азеотропов по данным о бинарных системах, экспериментально более доступным и широко представленным в литературе.

Число работ по предсказанию и расчету многокомпонентных азеотропов продолжает увеличиваться, что само по себе свидетельствует о настоятельной потребности заменить экспериментальное исследование расчетным, а также об отсутствии единого универсального метода, который позволил бы с высокой точностью рассчитывать параметры азеотропов произвольного типа. В дальнейшем изложении даны сравнительная характеристика и краткая оценка существующих методов расчета азеотропных свойств многокомпонентных, в том числе тройных систем.

Расчет характеристик азеотропного состояния является частью более общего моделирования фазового равновесия жидкость-пар и жидкость-жидкость-пар в многокомпонентной системе. Поэтому для предсказания азеотропных характеристик нашли применение методы расчета указанных фазовых равновесий и, в первую очередь, наиболее распространенные из них аналитические методы, основанные на эмпирической 
и полуэмпирической зависимости избыточной свободной энергии или коэффициентов активности от состава смеси. Уравнения этой зависимости будем в дальнейшем называть интерполяционными.

Во многих методах расчета используется сочетание соотношений для учета неидеальности жидкой фазы в форме уравнения концентрационной зависимости коэффициентов активности с поиском экстремальной или стационарной точки на поверхности температуры $(T)$ или давления $(P)$ многокомпонентной системы

$$
\begin{aligned}
& \left(\frac{\mathrm{d} T}{\mathrm{~d} x_{i}}\right)_{P}=0, i=1,2, \ldots, n-1, \\
& \left(\frac{\mathrm{d} P}{\mathrm{~d} x_{\mathrm{i}}}\right)_{T}=0, i=1,2, \ldots, n-1,
\end{aligned}
$$

где $x_{i}$ - молярная доля компонента $i$. Эта точка характеризуется равенством составов жидкости $\left(x_{i}\right)$ и пара $\left(y_{i}\right)$ :

$$
x_{i}=y_{i}, i=1,2, \ldots, n \text {. }
$$

Из условий равенства составов сосуществующих фаз (3) и идеального поведения пара получают выражения для коэффициента активности компонента

$$
\gamma_{i}=P / P_{i}^{0}
$$

где $P_{i}^{n}$ - давление пара компонента $i$, и отношения коэффициентов активности двух компонентов

$$
\gamma_{i} / \gamma_{k}=P_{k}^{0} / P_{i}^{0}
$$

в азеотропной точке. С помощью этих выражений могут быть рассчитаны давление пара и состав многокомпонентного азеотропа в изотермических условиях при использовании избранной формы интерполяционного уравнения и значений давлений паров компонентов. Для расчета состава азеотропа в изобарических условиях должна быть известна его температура кипения. В методах изобарического расчета температуру кипения азеотропа или заранее предполагают известной [3], или находят методом подбора $\left[{ }^{4}\right]$, или, наконец, учитывая условие (4), связывают с составом азеотропа путем введения выражений температурной зависимости давления пара компонентов в уравнение Скэтчарда для избыточной свободной энергии $\left(G^{E}\right)$

$$
G^{E}=R T \sum_{i} x_{i} \ln \gamma_{i}
$$

которое затем приравнивают к одному из интерполяционных уравнений $\left[{ }^{5-7}\right]$.

В ранних методах предсказания азеотропных характеристик тройных систем для выражения неидеальности жидкой фазы были использованы уравнения регулярных растворов $\left[{ }^{5,8}\right]$. Ряд более поздних методов основан на применении частных форм уравнения Вооля [9].

С усложнением форм корреляционных уравнений значительно увеличилась трудоемкость расчетов, в особенности расчетов в изобарических условиях, без заметного, однако, повышения точности получаемых результатов. Более того, можно привести немало примеров, когда простой по технике расчета метод Малесинского, основанный на модели регулярного раствора, приводит к более точным результатам расчета состава тройного азеотропа, чем методы, в основе которых лежат уравнения группы Вооля (табл. 1). 
Предсказание тройных азеотропов методами Малесинского [5] и Хорвата $\left.{ }^{4}\right]$

\begin{tabular}{|c|c|c|c|c|c|c|}
\hline \multirow{3}{*}{$\begin{array}{l}\text { Азеотроп } \\
\begin{array}{l}\text { Ацетон } \\
\text { хлороформ } \\
\text { метанол }\end{array}\end{array}$} & \multicolumn{2}{|c|}{ Эксперимент } & \multicolumn{4}{|c|}{ Расчет методом } \\
\hline & \multirow{2}{*}{$\begin{array}{c}T_{\text {кни }}, \mathrm{K} \\
330,6\end{array}$} & \multirow{2}{*}{$\begin{array}{l}x_{i} \\
0,315 \\
0,239 \\
0,446\end{array}$} & \multicolumn{2}{|c|}{ Малесинского * } & \multicolumn{2}{|c|}{ Хорвата } \\
\hline & & & 330,3 & $\begin{array}{l}0,364 \\
0,179 \\
0,457\end{array}$ & 329,9 & $\begin{array}{l}0,444 \\
0,196 \\
0,360\end{array}$ \\
\hline $\begin{array}{l}\text { Ацетон } \\
\text { метанол } \\
\text { циклогексан }\end{array}$ & 324,4 & $\begin{array}{l}0,438 \\
0,282 \\
0,280\end{array}$ & 324,6 & $\begin{array}{l}0,458 \\
0,270 \\
0,272\end{array}$ & 326,1 & $\begin{array}{l}0,478 \\
0,255 \\
0,267\end{array}$ \\
\hline $\begin{array}{l}\text { Бензол } \\
\text { вода } \\
\text { этанол }\end{array}$ & 338,01 & $\begin{array}{l}0,539 \\
0,233 \\
0,228\end{array}$ & 337,1 & $\begin{array}{l}0,573 \\
0,204 \\
0,233\end{array}$ & 341,1 & $\begin{array}{l}0,593 \\
0,072 \\
0,333\end{array}$ \\
\hline
\end{tabular}

* Оптимальный вариант трех результатов расчета состава.

К значительным ошибкам в составах тройных азеотропов привели также расчеты по уравнению Маргулеса [10]. В некоторых системах расчет вообще не выявил тройного азеотропа, а в других указал на два азеотропа, что также противоречило действительности.

K заметному повышению точности расчета азеотропных характеристик привело использование полуэмпирических интерполяционных уравнений, основанных на концепции локального состава [1']. Повышению точности способствовали также достижения вычислительной техники, позволившие применить для определения азеотропных характеристик машинный расчет, без которого решение столь сложных уравнений, как уравнения Вильсона, NRTL, UNIQUAC и других, основанных на представлении о локальном составе раствора, было бы немыслимым.

Благодаря ЭВМ стали возможными более совершенная оценка параметров уравнений с применением специальных целевых функций и методов оптимизации и более широкое внедрение методов итерации в расчет азеотропных характеристик. Вопрос о температуре кипения многокомпонентного азеотропа при постоянном давлении, например, успешно решается теперь путем итерационного расчета - до совпадения вычисленной и заданной величин общего давления с наперед установленной точностью. Определение состава азеотропа может быть сведено к понску минимума функций, связанных с азеотропными составами, например функций

$$
F \equiv \sum_{i=1}^{n-1}\left|\alpha_{i n}-1\right|, \alpha_{i n}=\frac{y_{i} x_{n}}{y_{n} x_{i}}
$$

и

$$
F \equiv \sum_{i=1}^{n}\left|k_{i}-1\right|, k_{i}=y_{i} / x_{i}
$$

с учетом того, что значение относительной летучести $\left(\alpha_{i n}\right)$ и коэффициента распределения $\left(k_{i}\right)$ в азеотропной точке, согласно условию (3), равны единице.

Применение ЭВМ способствовало проведению ряда сравнительных расчетов фазовых равновесий в многокомпонентных системах, которые в ряде случаев, например в [12], показали преимущество уравнений локального состава, в частности, уравнения Вильсона перед двухпараметрическими уравнениями группы Вооля. Хорошие результаты получены с помощью уравнения Вильсона также при расчете азеотропных характеристик тройных и многокомпонентных систем [11, 13-15]. 
При сравнительном расчете свойств тройных азеотропов по уравнениям Вильсона, NRTL и Редлиха-Кистера, выполненном А. Г. Морачевским и И. Б. Пукинским [1'], преимущество уравнения Вильсона проявилось даже в большей степени, чем при расчете равновесия жидкостьпар во всей области составов тройных систем. Точность расчета составов десяти тройных гомоазеотропов при использовании разной исходной информации для определения параметров названных уравнений оценена по значениям средних абсолютных расхождений с экспериментальными данными (табл. 2).

Таблица 2

Средняя абсолютная ошибка при расчете азеотропных составов тройных систем $\left.{ }^{11}\right]$, мол. \%

\begin{tabular}{l|c|c|c}
\hline \multirow{2}{*}{$\begin{array}{c}\text { Исходные данные для расчета } \\
\text { параметров уравнений }\end{array}$} & \multicolumn{3}{|c}{ Метод } \\
\cline { 2 - 4 } & Редлиха-Кистера & NRTL & Вильсона \\
\hline O бинарных растворах во всей области & 2,7 & 1,7 & 1,9 \\
составов & 2,0 & 2,2 & 1,9 \\
O бннарных эквимолярных растворах & 3,7 & 2,4 & 2,2 \\
О бинарных азеотропах & &
\end{tabular}

Bсе три уравнения были подвергнуты жесткой проверке: половина рассмотренных азеотропов принадлежала к типу почти тангенциальных, состав которых не всегда верно определяется расчетом. При всех трех вариантах расчета параметров уравнений применение уравнения Редлиха-Кистера указало на отсутствие тройного азеотропа в пяти системах. Расчет по уравнению NRTL, третья константа которого оценивалась априорно, не выявил азеотропа в одной системе (этанол-этилацетатциклогексан) при всех трех способах оценки параметров, а в другой (бензол-циклогексан-бутанол) - при оценке параметров по данным об эквимолярных и азеотропных растворах. Для всех исследованных систем только уравнение Вильсона дало качественно правильный результат при всех вариантах оценки параметров. Для практики весьма важно, что правильный результат получен и на основе данных о бинарных азеотропах, более распространенных в литературе, чем данные о равновесии жидкость-пар в бинарных системах.

В противовес сказанному в $\left[{ }^{16}\right]$ получено большее расхождение между экспериментом и расчетом по уравнению Вильсона, в том числе и для тех тройных азеотропов, которые ранее были предсказаны с высокой точностью (табл. 3).

Таблица 3

Предсказание составов $\left(x_{i}\right)$ тройных азеотропов с помощью уравнения Вильсона

\begin{tabular}{|c|c|c|c|c|c|c|c|}
\hline \multirow{2}{*}{ Азеотроп } & \multirow{2}{*}{\multicolumn{2}{|c|}{$\begin{array}{l}\text { Эксперимент при } \\
1013 \text { гПа, } 382 \mathrm{~K}\end{array}$}} & \multicolumn{5}{|c|}{ Расчет } \\
\hline & & & {$\left[{ }^{11}\right]$} & {$\left[{ }^{13}\right]$} & {$\left[{ }^{14}\right]$} & {$\left[{ }^{15}\right]$} & {$\left[{ }^{16}\right]$} \\
\hline $\begin{array}{l}\text { Метилацетат } \\
\text { хлороформ } \\
\text { Метанол }\end{array}$ & $\begin{array}{l}0,248 \\
0,293 \\
0,459\end{array}$ & $\bar{z}$ & $\begin{array}{l}0,257 \\
0,303 \\
0,440\end{array}$ & $\begin{array}{l}0,257 \\
0,291 \\
0,452\end{array}$ & $\begin{array}{l}0,265 \\
0,285 \\
0,450\end{array}$ & $\bar{z}$ & $\begin{array}{l}\text { азеотроп } \\
\text { не найдев }\end{array}$ \\
\hline $\begin{array}{l}\text { Ацетон } \\
\text { хлороформ } \\
\text { этанол }\end{array}$ & $\begin{array}{l}0,350 \\
0,460 \\
0,190\end{array}$ & $\begin{array}{l}0,35 \\
0,52 \\
0,13\end{array}$ & $\begin{array}{l}0,353 \\
0,469 \\
0,178\end{array}$ & $\overline{-}$ & $\begin{array}{l}0,345 \\
0,495 \\
0,160\end{array}$ & $\begin{array}{l}0,360 \\
0,464 \\
0,176\end{array}$ & $\begin{array}{l}0,231 \\
0,289 \\
0,480\end{array}$ \\
\hline
\end{tabular}


Расхождение данных $\left[{ }^{16}\right]$ с результатами расчетов $\left[{ }^{11,}{ }^{13-15}\right]$ вряд ли можно отнести за счет различия в параметрах уравнения и в способе расчета азеотропных составов, различающихся во всех упомянутых работах.

Значительное расхождение с экспериментом получено в [16] также при расчете характеристик тройных азеотропов по уравнению Вильсона с тройными константами, что также не согласуется с результатами $\left[{ }^{11,}{ }^{13}\right]$, которые свидетельствуют о прямой связи между точностью расчета и полнотой исходной информации. Аналогичный расчет с применением уравнения Редлиха-Кистера оказался несколько точнее [ $\left.{ }^{16}\right]$.

Заманчивая возможность расчета азеотропных характеристик по весьма ограниченному объему исходных данных кроется в групповых моделях [17]. Если известны параметры взаимодействия для всех пар структурных групп, образующих смесь, то ее азеотропные параметры могут быть рассчитаны без привлечения данных о смесях.

Хорошие результаты были получены с помощью групповой модели UNIFAC для четырех тройных гомоазеотропов в широком интервале давлений $\left[{ }^{18}\right]$. Среди них, в частности, азеотроп этилацетат-этанол-циклогексан, который не был выявлен расчетом ни с помощью уравнения NRTL, ни с помощью уравнения Редлиха-Кистера [1']. Расчет же по модели UNIFAC хорошо воспроизводит экспериментальные данные об изменении состава и температуры кипения этого азеотропа при изменении давления (табл. 4).

Таблица 4

Предсказание изменения состава и температуры кипения азеотропа этилацетат-этанол-циклогексан при изменении давления

\begin{tabular}{c|c|c|c|c|c|c|c|c}
\hline \multirow{2}{*}{$P$, гПа } & \multirow{2}{*}{$T, \mathrm{~K}$} & \multicolumn{3}{|c|}{ Эксперимент * } & \multicolumn{3}{|c}{ Расчет по модели UNIFAC } \\
\cline { 3 - 8 } & & $x_{1}$ & $x_{2}$ & $x_{3}$ & $T, \mathrm{~K}$ & $x_{1}$ & $x_{2}$ & $x_{3}$ \\
\hline & & & & & & & & \\
1066 & 339,2 & 0,074 & 0,425 & 0,501 & 339,3 & 0,096 & 0,403 & 0,501 \\
933 & 335,3 & 0,092 & 0,404 & 0,504 & 335,5 & 0,106 & 0,390 & 0,504 \\
800 & 331,1 & 0,110 & 0,385 & 0,505 & 331,3 & 0,117 & 0,375 & 0,508 \\
667 & 326,5 & 0,122 & 0,372 & 0,506 & 326,8 & 0,129 & 0,360 & 0,511 \\
533 & 320,7 & 0,150 & 0,337 & 0,513 & 321,0 & 0,146 & 0,340 & 0,514 \\
400 & 314,2 & 0,172 & 0,308 & 0,520 & 314,7 & 0,165 & 0,319 & 0,516
\end{tabular}

* Результаты экстраполяции и интерполяции экспериментальных данных $\left[{ }^{19}\right]$ на указанные давления.

Модель UNIFAC может с успехом применяться также для предсказания тройных гетероазеотропов $\left[{ }^{20}\right]$. Из числа известных интерполяционных уравнений для предсказания свойств гетероазеотропов могут быть использованы те, которые пригодны для описания равновесия жидкость-жидкость-пар. Перспективным для расчета гетероазеотропов является, например, уравнение NRTL, которое, как было показано выше, дает вполне удовлетворительные результаты и при расчете тройных гомоазеотропов.

Помимо рассмотренных выше методов расчета азеотропных параметров многокомпонентных смесей, остановимся на двух, в которых не используются интерполяционные уравнения. В методе Клейна $\left.{ }^{21}\right]$ предполагается, что концентрационная зависимость температуры конденсации пара тройной системы описывается уравнением типа

$$
T_{123}=C_{20} y_{1}^{2}+C_{11} y_{1} y_{2}+C_{02} y_{2}^{2}+C_{10} y_{1}+C_{01} y_{2}+C_{00}
$$


Константы уравнения (9) могут быть определены по данным о бинарных системах. Возможность предсказания тройных азеотропов по уравнению (9) мало изучена.

В методе, разработанном с участием автора настоящей статьи $\left[{ }^{22,}{ }^{23}\right]$, состав тройного азеотропа определяется решением системы уравнений

$$
x_{i(i k)}=\frac{x_{i}}{1-x_{j}}=x_{i}^{i k}+C_{i k j} x_{j}
$$

описывающих на треугольнике составов проекции кривых $\alpha_{i k}=1$. Точка пересечения этих кривых, берущих начало в точках составов $\left(x_{i}^{i k}\right)$ бинарных азеотропов, отвечает составу тройного азеотропа. Константы $C_{i k j}$ могут быть рассчитаны по бинарным данным, в том числе по данным о бинарных азеотропах:

для гомоазеотропов

$$
C_{i k j}=-\frac{g_{i}+\left(g_{i j}-g_{i}\right) / x_{j}^{i j}-g_{k}-\left(g_{k j}-g_{k}\right) / x_{i}^{k i}}{\left(g_{i}-g_{i k}\right) / x_{k}^{i k}-\left(g_{i k}-g_{k}\right) / x_{i}^{i k}},
$$

для гетероазеотропов

$$
C_{i k j}=-\frac{\left(g_{i j}-g_{i}\right) / x_{j}^{i j}-\left(g_{h j}-g_{k}\right) / x_{j}^{k j}}{\left(g_{i}-g_{i k}\right) / x_{k}^{i k}-\left(g_{i k}-g_{h}\right) / x_{i}^{i k}},
$$

где $g=T$ или $\lg P$, одиночный и двойной индекс относятся к компоненту и бинарному азеотропу соответственно.

\begin{tabular}{|c|c|c|c|c|c|c|c|c|}
\hline \multirow{3}{*}{ Азеотроп } & \multicolumn{2}{|c|}{ Эксперимент } & \multicolumn{6}{|c|}{ Расчет } \\
\hline & \multirow[b]{2}{*}{$x_{i}$} & \multirow{2}{*}{$T$ нли $P$} & \multicolumn{2}{|c|}{ NRTL } & \multicolumn{2}{|c|}{ UNIFAC } & \multicolumn{2}{|c|}{ наш метод } \\
\hline & & & $\overline{\Delta x} \times 100$ & $\Delta T(\delta P \%)$ & $\overline{\Delta x} \times 100$ & $\Delta T(\delta P \%)$ & $\overline{\Delta x} \times 100$ & $\Delta T(\delta P \%)$ \\
\hline Этилацетат & 0,082 & & \multirow{3}{*}{\multicolumn{2}{|c|}{$\begin{array}{c}\text { азеотроп } \\
\text { не } \\
\text { найден }\end{array}$}} & \multirow{3}{*}{1,2} & \multirow{3}{*}{0,1} & \multirow{3}{*}{4,8} & \multirow{3}{*}{0,1} \\
\hline $\begin{array}{l}\text { этанол } \\
\text { циклогексан }\end{array}$ & $\begin{array}{l}0,416 \\
0,502\end{array}$ & $\begin{array}{c}338,00 \mathrm{~K} \\
\text { при } 1013 \text { гПа }\end{array}$ & & & & & & \\
\hline Пропилацетат & 0,392 & & & & & & & \\
\hline $\begin{array}{l}\text { пропанол } \\
\text { вода }\end{array}$ & $\begin{array}{l}0,091 \\
0,517\end{array}$ & $\begin{array}{c}355,60 \mathrm{~K} \\
\text { при } 1013 \text { гПа }\end{array}$ & 2,2 & 0,1 & \multirow{2}{*}{\multicolumn{2}{|c|}{$\begin{array}{c}\text { азеотроп } \\
\text { не } \\
\text { найден }\end{array}$}} & 1,9 & 0,1 \\
\hline 1,2-Дихлорэтан & 0,572 & & & & & & & \\
\hline $\begin{array}{l}\text { этанол } \\
\text { вода }\end{array}$ & $\begin{array}{l}0,209 \\
0,219\end{array}$ & $\begin{array}{c}312 \text { гПа } \\
\text { при } 313,2 \mathrm{~K}\end{array}$ & 4,6 & 6,2 & 2,4 & 4,9 & 2,6 & 1,1 \\
\hline$"$ & $\begin{array}{l}0,535 \\
0,230 \\
0,235\end{array}$ & $\begin{array}{c}756 \mathrm{rПa} \\
\text { при } 333,2 \mathrm{~K}\end{array}$ & 4,1 & 0,8 & 2,3 & 2,1 & 0,5 & 2,3 \\
\hline
\end{tabular}

Таблица 5

Предсказание тройных азеотропов

О бозн ачен и я. $\overrightarrow{\Delta x}$ и $\Delta T-$ среднне абсолютные ошибки расчета состава и температуры кипения азеотропа соответственно; $\delta P$ - относительная ошибка расчета давления пара азеотропа.

Точность расчета по уравнению (10) того же порядка, что и при расчете по методам NRTL и UNIFAC. Более надежно предсказываются, как правило, азеотропы с малым содержанием одного из компонентов (табл. 5). Эти три метода предсказывают свойства гетероазеотропов по данным о бинарных системах (метод NRTL), о бинарных азеотропах (наш метод) и о чистых компонентах (метод UNIFAC) не менее точно, чем эмпирическое уравнение Тамира $\left[{ }^{24}\right]$

$$
P(T)=\sum_{i=1}^{n} y_{i} P_{i}^{0}\left(T_{i}\right)+P^{E}\left(T^{E}\right),
$$


в котором неидеальность паровой фазы $\left[P^{E}\left(T^{E}\right)=f(y)\right]$ учитывается уравнением Редлиха-Кистера с тройными константами.

Решение системы уравнений (10) дает в общем случае три результата расчета (в табл. 5 дано их усредненное значение), что однако не указывает на образование трех тройных азеотропов в системе. Расхождение этих результатов, как правило, невелико и его причиной является отклонение истинных кривых $\alpha_{i k}=1$ от описываемых уравнением (10).

В заключение следует отметить, что образование нескольких азеотропов всеми компонентами системы не противоречит термодинамической теории, но встречается в действительности весьма редко. Поэтому каждый факт выявления расчетом нескольких азеотропов требует тщательной проверки. В тройной системе с этой целью можно воспользоваться анализом экспериментальных данных о составах сосуществующих жидкости и пара, представленных в форме изолиннй, связанных с азеотропами, например линий $\alpha_{i k}=1$ (рисунок). Ход изолиний $\alpha_{i k}=1$ в рассматриваемой здесь системе бензол-циклогексан-изопропанол (рисунок, $a$ ), выявленных по данным о равновесии жидкость-пар $\left[{ }^{25}\right]$, свидетельствует об образовании одного тройного азеотропа в отличие от результатов расчета $\left[{ }^{26}\right]$. Несмотря на существование двух бинарных азеотропов в системе бензол-перфторбензол, два названных компонента образуют с метилциклогексаном также только один тройной азеотроп $\left[{ }^{27}\right]$ (рисунок, б). Система гептан-изооктан-этанол (рисунок, в) представляет собой предельный случай часто встречающихся в практике тройных систем, в которых третий компонент оказывает слабое разделяющее дейст-

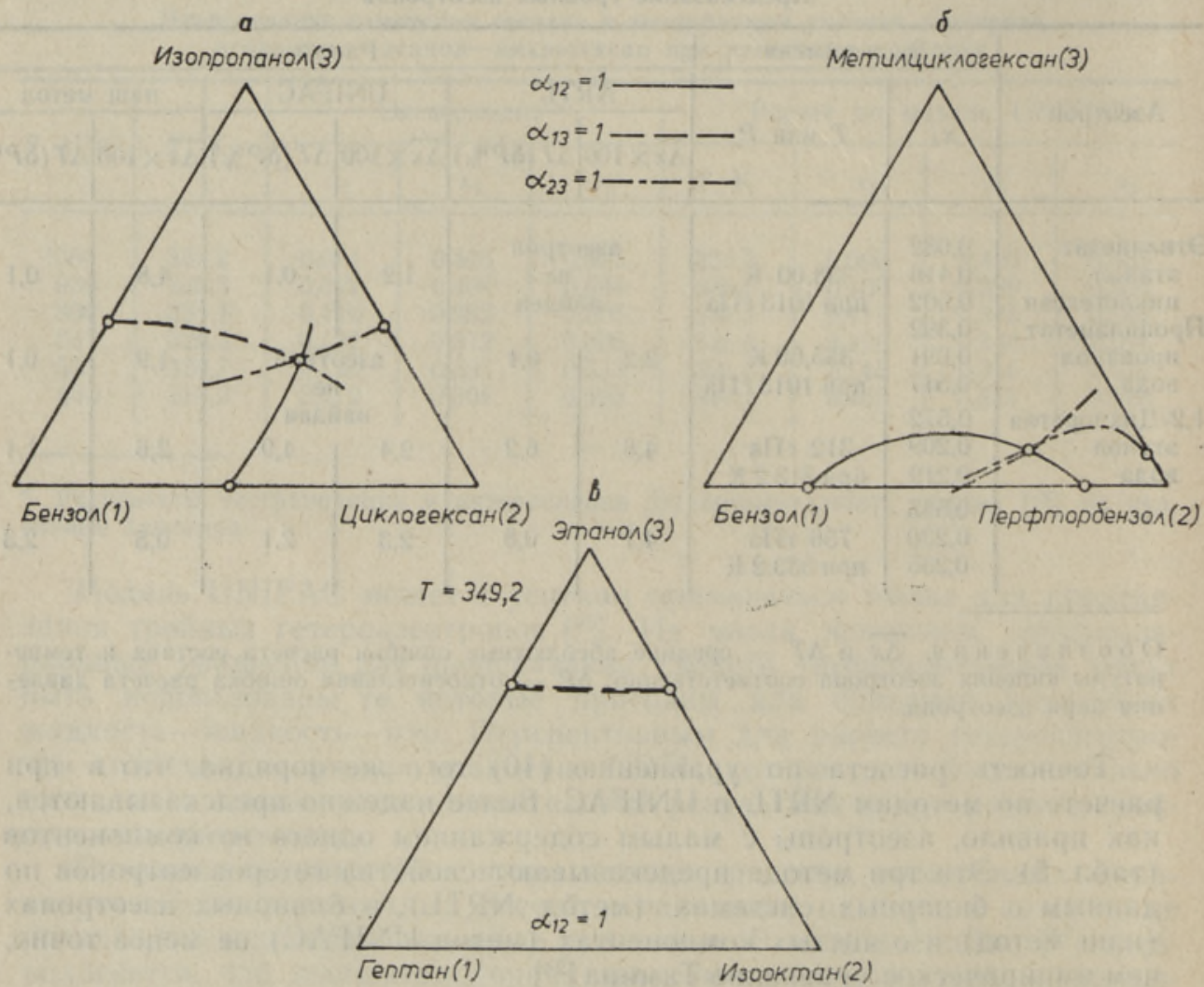

Расположенне линий $\alpha_{i k}=1$ на треугольниках составов тройных систем; о - состав азеотропа. 
вие на смеси двух других компонентов. Если эти компоненты не образуют азеотропа и кривая $\alpha_{12}=1$ не принадлежит треугольнику составов, то две другие кривые $\alpha_{i 3}=1 \quad(i=1,2)$ идут почти параллельно и близко друг от друга, в результате чего образуется полоса составов, характеризующихся короткими нодами. В системе 8 при указанной температуре компоненты идеальной смеси гептан (1) - изооктан (2) имеют одинаковые давления паров, вследствие чего их относительная летучесть во всем концентрационном интервале бинарной системы равна единице. В пределах погрешности эксперимента это значение $\alpha_{12}$ сохраняется и во всей концентрационной области тройной системы. Кривые $\alpha_{13}=1$ и $\alpha_{23}=1$ слились в общую кривую, каждую точку которой пересекают кривые $\alpha_{12}=1$. В тройной системе образуется азеотроп, который можно назвать «азеотропом переменного состава» $\left[{ }^{28}\right]$.

\section{ЛИТЕРАТУРА}

1. Коган В. Б. Азеотропная и экстрактивная ректификация. Л., 1972.

2. Жаров В. Т., Серафимов Л. А. Физико-химические основы дистилляции и ректифнкацин. Л., 1975.

3. Hollo, J., Lengyel, T. Vapor-liquid equilibrium of the system toluene-pyridine1-butanol. - Ind. Eng. Chem., 1959, 51, N 8, 957-960.

4. Horvath, P. J. Graphical prediction of ternary azeotropes. - Chem. Eng., 1961, 68, N 6, 159-164.

5. Malesinski, W. Azeotropy and Other Theoretical Problems of Vapour-liquid Equilibrium. New York, 1965.

6. Yamada, J., Yoshida, T., Kawase, $O$. Analysis of the multiazeotropes in terms of the zeroth approximation. - Bull. Chem. Soc. Jap., 1959, 32, 570-573.

7. Raju, B. N., Rao, M. N. A method for the accurate prediction of binary and ternary azeotropes at atmospheric pressure. - Indian Chem. Eng., 1966, 8, N 2, $21-27$.

8. Haase, R. Verdampfungsgleichgewichte von Mehrstoffgemischen. 7. Mitt. Ternäre azeotrope Punkte. $-Z$. phys. Chem., 1950, 195, 362-385.

9. Хала Э., Пик И., Фрид В., Вилим О. Равновесие между жидкостью и паром. М., 1962 .

10. Мариничев А. Н., Виниченко Н. Г. О возможности исследования азеотропных свойств тройных систем с помощью методов расчета изотермического равновесия жидкость-пар. - Ж. прикл. хим., 1969, 43, № 3, 558-563.

11. Grundlagen der Verfahrenstechnik und chemischen Technologie. Modellierung von Phasengleichgewichten als Grundlage von Stofftrennprozessen. Berlin, 1981

12. Holmes, M. J., van Winkle, M. Prediction of ternary vapor-liquid equilibria from binary data. - Ind. Eng. Chem., 1970, 62, N 1, 21 A-31A.

13. Нагата Н. Расчет данных по равновесию жидкость-пар в бинарных и тройных системах с помощью уравнения Вильсона. - Қагаку когаку, 1969, 33, № 3, 263-267. (Перевод ВИНИТИ.)

14. Аристович В. Ю., Степанова Э. Н. Расчетное определение многокомпонентных азеотропов по данным бинарных систем. - Ж. прикл. хим., 1970, 43, № 10, $2192-2200$.

15. Стяжкин В. Н., Кафаров В. В., Ветохин В. Н. Расчет азеотропных составов в многокомпонентных системах. - Химня и хим. технол., 1978, 21, № 9, 1319-1321.

16. Tamir, $A$. New correlations for fitting multicomponent vapor-liquid equilibria data and prediction of azeotropic behaviour. - Chem. Eng. Sci., 1981, 36, N 9, 14531465 .

17. Смирнова Н. А. Групповые модели раствора. - В кн.: Химия и термодинамика растворов, вып. 5. Л., 1982, $87-127$.

18. Skjold-Jorgensen, S., Kolbe, B., Gmehling, J., Rasmussen, $P$. Vapor-liquid equilibria by UNIFAC group-contribution. Revision and extension. - Ind. Eng. Chem. Process Des. Develop., 1979, 18, N 4, 714-722.

19. Сторонкин А. В., Морачевский А. Г., Кудрявцева Л. С. О влиянии температуры и давления на состав тройных азеотропов. - Ж. физ. хим., 1957, 31, № 2, 395-402.

20. Викторов А. И., Пукинский И. Б. Расчет равновесия жидкость-жидкость-пар в тройных системах. III. Всесоюзная школа «Применение математических методов для описания и изучения физико-химических равновесий». Тезисы докладов. Ч. 1. Новосибирск, $1980,151-155$. 
21. Klein, I. How to predict ternary azeotropes. - Chem. Eng., 1960, 67, N 11, 233234.

22. Kudrjawzewa, L. S., Toome, M. J., Sussarew, M. P. Zur Berechnung, der Zusammensetzung der ternären Azeotrope. - Monatsh. Chem., 1974, 105, N 3-4, 334339.

23. Кудрявцева Л. С. Расчет параметров многокомпонентных азеотропов. -Ж. прикл. хим., 1977,40 , № 5, 1075-1080.

24. Tamir, A. Correlation of vapor-liquid equilibria in systems showing homoazeotropy or heteroazeotropy using expressions for the total pressure and temperature as direct functions of vapor composition. - Chem. Eng. Sci., 1981, 36, N 9, 1467-1473.

25. Сторонкин А. В., Морачевский А. Г. О равновесии жидкость-пар в системе бензол-циклогексан-изопропиловый спирт. - Ж. физ. хим., 1956, 30, № 6, $1297-1307$.

26. Tamir, A., Wisniak, J. Correlation and prediction of boiling temperatures and azeotropic conditions in multicomponent systems. - Chem. Eng. Sci., 1978, $33, \mathrm{~N} 6,657-672$.

27. Wade, J. C., Taylor, Z. L., Jr. Vapor-liquid equilibrium in perfluorobenzene-benzene-methylcyclohexane system. - J. Chem. Eng. Data, 1973, 18, N 4 $424-427$.

28. Кудрявцева Л. С., Вийт $X^{\prime}$ Х., Эйзен О. Г. Равновесие жндкость-пар в тройных ситемах этанол-гептан-изооктан и гексен-1-гексан-этанол, - Ж. физ. хим., 1971, 45, № 7, 1859. Деп. ВИНИТИ, № 2810-71.

Институт химии

Академии наук Эстонской ССР
Поступила в редакцию 25/IX 1985

\section{KUDRJAVTSEVA}

\section{MITMEKOMPONENDILISTE ASEOTROOPIDE ENNUSTUSMEETODID}

Aseotroopide omaduste arvutamiseks (see kujutab endast osa üldisemast faasidevahelise tasakaalu modelleerimise ülesandest) osutuvad sobivaks komponentide aktiivsustegurite kontsentratsioonisōltuvuse võrrandid. Nende hulgas on eelistatumad poolempiirilised lokaalse koostise vôrrandid ja grupimudel UNIFAC. Täpsuse poolest voorreldavaid tulemusi saadakse ka mōningate spetsiaalsete meetoditega, mille väljatöötamisel on arvestatud aseotroopseid tingimusi. Olevaates on võrreldud kolmikaseotroopide ennustamise tulemusi.

\section{KUDRJAWZEWA}

\section{METHODEN ZUR VORAUSSAGE DER MEHRSTOFFAZEOTROPE}

Zur Azeotropeberechnung, die als ein Teil der allgemeineren Aufgabe des Modellierens der Phasengleichgewichte zu erfassen ist, eignen sich die Aktivitätskoeffizientengleichungen. Der Vorzug ist gegenwärtig den halbempirischen Gleichungen mit lokalen Brüchen und dem UNIFAC-Gruppenlösungsmodell zu geben. Vergleichbare Ergebnisse werden auch mit den für den azeotropen Zustand speziell entwickelten Vorausberechnungsmethoden gewonnen. Es werden einige Beispiele für ternäre Azeotrope zusammengestellt. 